\title{
Second-order Lagrangians admitting a first-order Hamiltonian formalism
}

\author{
M. Eugenia Rosado María \\ Departamento de Matemática Aplicada \\ Escuela Técnica Superior de Arquitectura, UPM \\ Avda. Juan de Herrera 4, 28040-Madrid, Spain
}

May 10, 2011

Let $p: E \rightarrow N$ be an arbitrary fibred manifold over a connected $n$ dimensional manifold $N$ oriented by a volume form $v=d x^{1} \wedge \cdots \wedge d x^{n}$, and let $p^{k}: J^{k} E \rightarrow N$ be the bundle of $k$-jets of local sections of $p$, with projections $p_{l}^{k}: J^{k} E \rightarrow J^{l} E$ for every $k \geq l$. Every fibred coordinate system $\left(x^{j}, y^{\alpha}\right)$ on $E$ for the projection $p, 1 \leq j \leq n, 1 \leq \alpha \leq m=\operatorname{dim} E-\operatorname{dim} N$, induces a coordinate system $\left(x^{j}, y_{I}^{\alpha}\right)$, on the $r$-jet bundle, where $I=\left(i_{1}, \ldots, i_{n}\right) \in \mathbb{N}^{n}$ is an integer multi-index of order $|I|=i_{1}+\cdots+i_{n} \leq r$; namely,

$$
y_{I}^{\alpha}\left(j_{x}^{r} s\right)=\frac{\partial^{|I|}\left(y^{\alpha} \circ s\right)}{\partial\left(x^{1}\right)^{i_{1}} \cdots \partial\left(x^{n}\right)^{i_{n}}}(x),
$$

where $s$ is a local section of $p$ defined on a neighbourhood of $x \in N$. We use the notations $I=(j)=(0, \ldots, 0, \stackrel{(j}{1}, 0, \ldots, 0) \in \mathbb{N}^{n}$ and $y_{(j)}^{\alpha}=y_{j}^{\alpha}$.

The Legendre form of a second-order Lagrangian density $\Lambda=L v$ defined on $p: E \rightarrow N$, where $L \in C^{\infty}\left(J^{2} E\right)$, is the $V^{*}\left(p^{1}\right)$-valued $p^{3}$-horizontal $(n-1)$-form $\omega_{\Lambda}$ on $J^{3} E$ is locally given by (e.g., see $[3,5]$ ),

$$
\omega_{\Lambda}=(-1)^{i-1} L_{\alpha}^{i 0} v_{i} \otimes d y^{\alpha}+(-1)^{i-1} L_{\alpha}^{i(j)} v_{i} \otimes d y_{j}^{\alpha},
$$

where $v_{i}=d x^{1} \wedge \cdots \wedge \widehat{d x^{i}} \wedge \cdots \wedge d x^{n}$, and

$$
\begin{aligned}
L_{\alpha}^{i(j)} & =\frac{1}{2-\delta_{i j}} \frac{\partial L}{\partial y_{(i j)}^{\alpha}}, \\
L_{\alpha}^{i 0} & =\frac{\partial L}{\partial y_{i}^{\alpha}}-\frac{1}{2-\delta_{i j}} D_{j}\left(\frac{\partial L}{\partial y_{(i j)}^{\alpha}}\right),
\end{aligned}
$$

where $D_{j}$ denotes the "total derivative" with respect to the coordinate $x^{j}$, i.e.,

$$
D_{j}=\frac{\partial}{\partial x^{j}}+\sum_{|I|=0}^{\infty} \sum_{\alpha=1}^{m} y_{I+(j)}^{\alpha} \frac{\partial}{\partial y_{I}^{\alpha}}
$$


The Poincaré-Cartan form attached to $\Lambda$ is then defined to be the ordinary $n$-form on $J^{3} E$ given by (e.g., see [3], [5]),

$$
\Theta_{\Lambda}=\left(p_{2}^{3}\right)^{*} \theta^{2} \wedge \omega_{\Lambda}+\Lambda
$$

where $\theta^{2}$ is the second-order structure form on $J^{2} E$ locally given in coordinates as follows (cf. [2], [4]):

$$
\theta^{2}=\left(d y^{\alpha}-y_{i}^{\alpha} d x^{i}\right) \otimes \frac{\partial}{\partial y^{\alpha}}+\left(d y_{h}^{\alpha}-y_{(h i)}^{\alpha} d x^{i}\right) \otimes \frac{\partial}{\partial y_{h}^{\alpha}}
$$

and the exterior product of $\left(p_{2}^{3}\right)^{*} \theta^{2}$ and the Legendre form, is taken with respect to the pairing induced by duality, $V\left(p^{1}\right) \times_{J^{1} E} V^{*}\left(p^{1}\right) \rightarrow \mathbb{R}$.

The most outstanding difference with a first-order Lagrangian density is that the Legendre and Poincaré-Cartan forms associated with a secondorder Lagrangian density are generally defined on $J^{3} E$, thus increasing by one the order of the Lagrangian density $\Lambda$.

For certain second-order Lagrangian densities it is well known that the Poincaré-Cartan form is projectable onto $J^{2} E$; e.g., see [1]. More precisely, the Poincaré-Cartan form of a second-order Lagrangian projects onto $J^{2} E$ if and only if the following system of PDEs holds (cf. [1]):

$$
\frac{1}{2-\delta_{i b}} \frac{\partial^{2} L}{\partial y_{a c}^{\beta} \partial y_{i b}^{\alpha}}+\frac{1}{2-\delta_{i a}} \frac{\partial^{2} L}{\partial y_{b c}^{\beta} \partial y_{i a}^{\alpha}}+\frac{1}{2-\delta_{i c}} \frac{\partial^{2} L}{\partial y_{a b}^{\beta} \partial y_{i c}^{\alpha}}=0,
$$

for all indices $1 \leq a \leq b \leq c \leq n, \alpha, \beta=1, \ldots, m$.

More surprisingly, there exist second-order Lagrangians for which the associated Poincaré-Cartan form projects not only on $J^{2} E$ but also on $J^{1} E$. Notably, this is the case of the Einstein-Hilbert Lagrange in General Relativity.

In this talk we obtain a characterization of such Lagrangians and we study its Hamiltonian formalism.

\section{References}

[1] Pedro L. García, J. Muñoz Masqué, Le problème de la régularité dans le calcul des variations du second ordre, C.R. Acad. Sci. Paris 301 Série I (1985), 639-642.

[2] J. Muñoz Masqué, Formes de structure et transformations infinitésimales de contact d'ordre supérieur, C.R. Acad. Sci. Paris 298, Série I (1984), $185-188$.

[3] -, An axiomatic characterization of the Poincaré-Cartan form for second-order variational problems, Lecture Notes in Math. 1139, Springer-Verlag 1985, pp. 74-84. 
[4] D. J. Saunders, The Geometry of Jet Bundles, Cambridge University Press, Cambridge, UK, 1989.

[5] D. J. Saunders, M. Crampin, On the Legendre map in higher-order field theories, J. Phys. A: Math. Gen. 23 (1990), 3169-3182. 